\title{
Diseño y prototipo de un precipitador electrostático
}

\section{Design and prototype of an electrostatic precipitator}

\author{
Juan Felipe Rodríguez \\ Semillero s1 Ro, Universidad de Bogotá Jorge Tadeo Lozano, Bogotá, D. C., Colombia \\ http://orcid.org/0000-0001-8105-1616 \\ Uriel Faruk Alarcón Pinto \\ Semillero s1 Ro, Universidad de Bogotá Jorge Tadeo Lozano, Bogotá, D. C., Colombia \\ http://orcid.org/0000-0002-1990-024X \\ Olmer García Bedoya \\ Profesor departamento de Ingeniería Universidad de Bogotá Jorge Tadeo Lozano, Bogotá, D. C., Colombia \\ http://orcid.org/0000-0002-6964-3034 \\ olmer.garciab@utadeo.edu.co \\ Germán Benavides \\ TadeoLab, Universidad de Bogotá Jorge Tadeo Lozano, Bogotá, D. C., Colombia \\ http://orcid.org/0000-0002-3055-4384
}

Fecha de recepción: 13 de junio de 2017 Fecha de aceptación: 08 de agosto de 2017

Sugerencia de citación: Rodríguez, J. F., Alarcón, U. F., García Bedoya, O. y Benavides, G. (2017). Diseño y prototipo de un precipitador electrostático. Mutis, 7(2), 86-95, doi: http://dx.doi.org/10.21789/22561498.1237

Editor: Díaz, C. cesaro.diazb@utadeo.edu.co

\section{RESUMEN}

En vista del alto grado de contaminación que se presenta hoy en día, se han comenzado a buscar medidas para combatir y regular este problema. Entre estas medidas están los precipitadores electrostáticos (PES), dispositivos usados en el proceso de purificar el medio ambiente. Este proceso se realiza por medio de alta tensión de voltaje en placas de metales para generar campos eléctricos que atrapan las partículas de contaminantes. De esta manera se purifica el aire del ambiente. Para el proyecto se utiliza un circuito que aumenta la tensión de la red local (120 V) a 27000 voltios por medio de un transformador conocido como flyback. Este proyecto busca diseñar y construir un dispositivo de este tipo con elementos de fácil adquisición, con la finalidad de entender cómo puede ser usado en hogares y zonas urbanas. Se logró realizar un prototipo eficaz para limpiar el aire con elementos y materiales de bajo costo.

Palabras clave: Arduino, precipitador electrostático, flyback, calidad del aire. 


\section{ABSTRACT}

In view of the high degree of contamination that occurs today, they have begun to seek measures to combat and regulate this problem. One of these measures are electrostatic precipitators (ESPS), used in the process of purifying the environment by means of high voltage stress plates metals to generate electric fields which trap contaminant particles purifying thus air devices ambient. Project a circuit for increasing the voltage of the local network (120 V) to 27000 volts by a transformer, known as flyback used. The purpose of this project is to demonstrate the efficacy of this device, and how it can be built with economical and affordable materials for deployment in homes and urban areas. It was possible to raise an effective air cleaning elements and materials with low-cost prototype.

Keywords: Arduino, electrostatic precipitator, flyback, air quality.

\section{INTRODUCCIÓN}

Según la Organización Mundial de la Salud, Bogotá, una de las principales ciudades de Colombia, es una de las que tienen peor calidad de aire. En 2014, según esta entidad, Bogotá presentaba una concentración de partículas de PM 10 de 48 microgramos por metro cúbico, que se considera casi el triple del tope internacional. Las altas tasas de contaminación en el aire han obligado a dictar medidas como la de no recomendar el ejercicio en zonas abiertas en ciudades como Shanghái, Ciudad de México o Medellín. Esto ha impulsado la búsqueda de diferentes técnicas para controlar y atacar la generación de la contaminación. Entre ellas están los precipitadores electrostáticos, que son ampliamente utilizados en la industria. En este proyecto se busca diseñar y probar un dispositivo de este tipo con elementos de fácil adquisición con la finalidad de entender cómo puede ser usado en zonas urbanas como casas y oficinas.

En Colombia, el Ministerio de Ambiente, Vivienda y Desarrollo Territorial, a finales del año 2010 creó un protocolo para controlar y vigilar la contaminación (MAVDT, 2010). En él, como respuesta al artículo 72 de la Resolución 909 del 5 de junio de 2008 (Resolución $909,2008)$, se evaluó y se estudió la implementación de sistemas para ayudar a controlar la contaminación en casos de fuentes fijas de contaminación, por medio de precipitadores electroestáticos. Según la Resolución 909 del Ministerio de Ambiente, Vivienda y Desarrollo Territorial, las fuentes fijas de contaminación se pueden clasificar, de acuerdo con la envergadura y distribución espacial de las emisiones, en fuentes fijas puntuales y fuentes fijas difusas o dispersas.

La precipitación electrostática de las moléculas se dio a conocer en los primeros años del siglo Xx. Desde esa época su aplicación en las industrias ha ido creciendo. En la actualidad es uno de los procesos de eliminación de moléculas más empleados en las industrias. Como es presentado en Sokhi (2005), el principio del precipitador electrostático fue desarrollado por el físico y químico estadounidense Frederick Gardner Cottrell, profesor de la Universidad de California, en Berkley, quien en 1906 también desarrolló el primer precipitador electrostático (PES) comercial, exitoso, que fue instalado en una planta manufacturera de ácido cerca de Pinole, California.

En la actualidad existen varios tipos de PES:

Precipitador placa-alambre: es un tipo de precipitador utilizado principalmente por industrias que hacen uso del calor, como las calderas que queman el carbón, los hornos de cemento, hornos de chimenea abierta, etc.

Precipitador tubular o tubo-alambre: lOS PES originales eran tubulares, como las chimeneas, donde eran colocados, con los electrodos a alto voltaje orientados a lo largo del eje del tubo. Los precipitadores tubulares tienen aplicaciones típicas en plantas de adición de ácido sulfúrico, limpieza del gas subproducto de los hornos de coque (remoción de alquitrán) y, recientemente, plantas de sinterización de hierro y acero. Tales unidades tubulares aún son utilizadas para 
algunas aplicaciones; suelen tener muchos tubos en paralelo para manejar mayores flujos de gas. Los tubos pueden tener forma de panal circular, cuadrado o hexagonal, y el gas fluye hacia arriba o hacia abajo (Turner, Lawless, Yamamoto y Coy Turner, 1999).

Precipitador de placas planas: utiliza placas como reemplazo de alambres para los electrodos expuestos al alto voltaje. Las placas planas ayudan a intensificar el campo eléctrico, que se usa para la recolección de partículas; también aporta una mayor área para la recolección de estas.

Precipitador húmedo (PEH): recibe este nombre debido a que cualquiera de los otros tipos ya mencionados se puede configurar para que se reemplacen las paredes secas o placas por paredes húmedas. LOS PEH se utilizan en situaciones en las que los precipitadores electrostáticos secos (PES) no son apropiados, cuando el material por recolectar está húmedo, pegajoso, es inflamable, es explosivo o tiene una resistividad alta (Fernández, 2012).
El presente artículo, primero, presenta una descripción de los principios de funcionamiento del PES; segundo, muestra el proceso de diseño y desarrollo del prototipo de placas planas; tercero, indica los resultados experimentales del prototipo por medio de mediciones de contaminación a la entrada y a la salida, y, finalmente, presenta las conclusiones y los trabajos futuros.

\section{EL PRECIPITADOR ELECTROESTÁTICO}

El principio de ionización es la base del funcionamiento de un PES, es decir, las partículas que pasan a través de las placas se ven afectadas por un campo eléctrico, lo que hace que estas se carguen eléctricamente. Los gases pasan por el interior de una cámara, lugar donde se crea el campo electrostático formado por electrodos de descarga (negativo) y electrodos colectores (positivo); ambos electrodos son de placas de cobre. Cuando pasa el gas por las placas, este se carga negativamente debido al principio de ionización, y es atraído a los electrodos colectores debido a su diferente carga eléctrica (figura 1). A continuación se presentan los conceptos físicos utilizados para el diseño del dispositivo.

Figura 1. Principio del precipitador electroestático

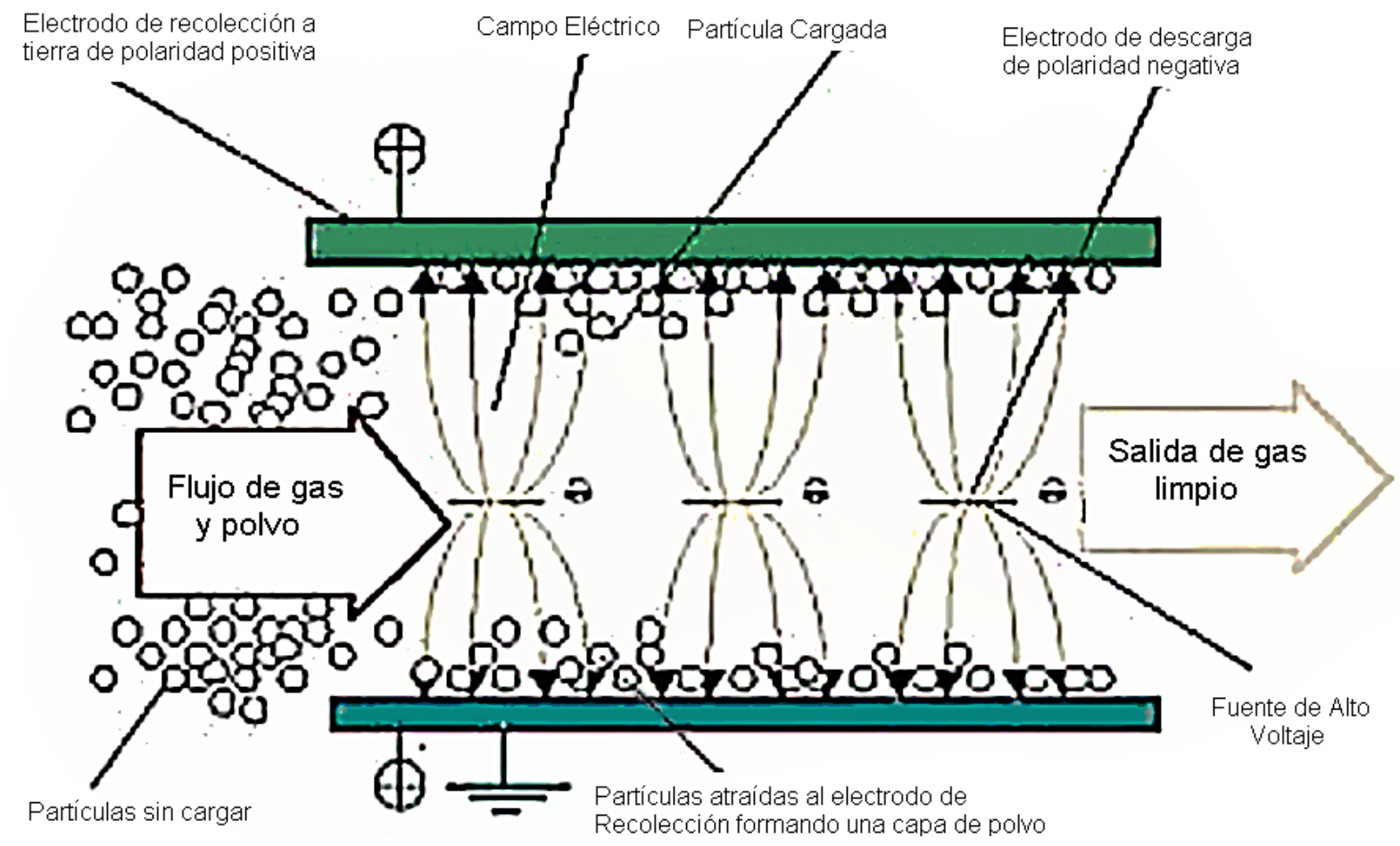

Fuente: Guerrero, 2014 


\section{A. Velocidad de deriva}

La velocidad de deriva $w_{e}$ es el resultado del balance entre la fuerza electrostática, gracias a la carga $F_{e^{\prime}}$ y la fuerza de arrastre, $F_{d^{\prime}}$ ejercida por el aire debido a la relación del movimiento entre el aire y las partículas:

$$
\begin{aligned}
& E=\frac{\Delta V}{d} \\
& F_{e}=\text { carga } \times \text { campo eléctrico }=q E \\
& F_{d}=\text { fuerza de arrastre }=\frac{3 \pi \mu_{f} d_{p} w_{e}}{C_{c}} \\
& \qquad F_{d=} F_{e \rightarrow} \frac{3 \pi \mu_{f} a_{p} w_{c}}{c_{c}}=q E=w_{e}=\frac{c_{c} q E}{3 \pi \mu_{f}}
\end{aligned}
$$

Donde:

$$
\begin{aligned}
& \mathrm{Q}=\text { carga de cada partícula } \\
& \mathrm{E}=\text { campo eléctrico } \\
& C_{c}=\text { factor Cunningham } \\
& \mu_{f}=\text { viscosidad del fluido }=1.81 \times 10^{-5} \mathrm{~kg} / \mathrm{ms} \\
& \quad \text { por aire a temperatura ambiente } \\
& d_{P}=\text { diámetro de la partícula }
\end{aligned}
$$

La carga $q$ adquirida por la molécula, que es $1.6 \times 10^{-19}$ C (coulomb). Por ejemplo, la carga de una partícula que adquiere 10 electrones sería:

$$
q=10 \times 1.6 \times 10^{-19} \mathrm{C}=1.92 \times 10^{-18} \mathrm{C}
$$

El número de electrones adquiridos depende de la intensidad de la corona que se genera cerca de los electrones y, a la vez, es proporcional al campo eléctrico. De esta forma, es proporcional a lo que hace que la fuerza eléctrica sea proporcional a $E^{2}$.

Esta es la fórmula para determinar la carga adquirida por una partícula:

$$
q=\pi d_{p}^{2} \varepsilon_{0} \frac{3 \varepsilon}{2+\varepsilon} E_{c h}
$$

Donde

$\varepsilon 0=8,854 \times 10^{-12} C^{2} / V^{2} .=$ permitividad del vacío

$\varepsilon=3.7=$ constante dieléctrica de la partícula respecto al vacío

$E_{c h}=$ carga de la intensidad del campo en $(\mathrm{V} / \mathrm{m})$

\section{B. Rendimiento}

El rendimiento o eficacia es definido como el porcentaje de eliminación de partículas. Se puede hallar con la siguiente fórmula:

$$
\begin{aligned}
& n=\frac{\text { Partículas removidas }}{\text { Partículas de entrada }}=\frac{C_{\text {in }-} C_{\text {out }}}{C_{\text {in }}}=1-\frac{C_{\text {out }}}{C_{\text {in }}} \\
& =1-\exp \left(-\frac{w_{e} L}{U d}\right) \text { (3) }
\end{aligned}
$$

Dado que la velocidad de flujo $U$ es el flujo volumétrico $Q$ dividido por la sección de área transversal $W_{d}$, también se puede definir la eficacia así:

$n=1-\exp \left(-\frac{w_{c} W_{L}}{Q}\right)=1-\exp \left(-\frac{w_{c} A}{Q}\right)(4)$,

donde el área de las placas es el producto del largo por el ancho: $A=W L$.

Las anteriores fórmulas nos permitieron determinar que la capacidad del PES depende del tamaño de las placas, la separación entre ellas y el voltaje, que debe ser seleccionado de acuerdo con el tamaño y tipo de las partículas.

\section{DISEÑO Y CONSTRUCCIÓN DEL PRECIPITADOR}

El PES, como fue mostrado en la sección anterior, depende del campo eléctrico, que es proporcional al voltaje aplicado en las placas, al tipo de contaminante y a la geometría del mismo. A continuación se presenta cómo fue diseñada la fuente de alimentación, la fuente contaminante de pruebas y, finalmente, el diseño físico del dispositivo.

\section{A. Fuente de alimentación eléctrica}

Se escogió como fuente de alimentación un flyback, que es un convertidor DC a DC con aislamiento galvánico entre entrada y salida, que es capaz de almacenar toda la energía en el núcleo magnético. El modelo de este convertidor es FC20a25 (figura 2), común en los televisores de rayos catódicos. Este maneja un voltaje de salida de 27000 voltios. 
Figura 2. Modelo de flyback ${ }^{1}$

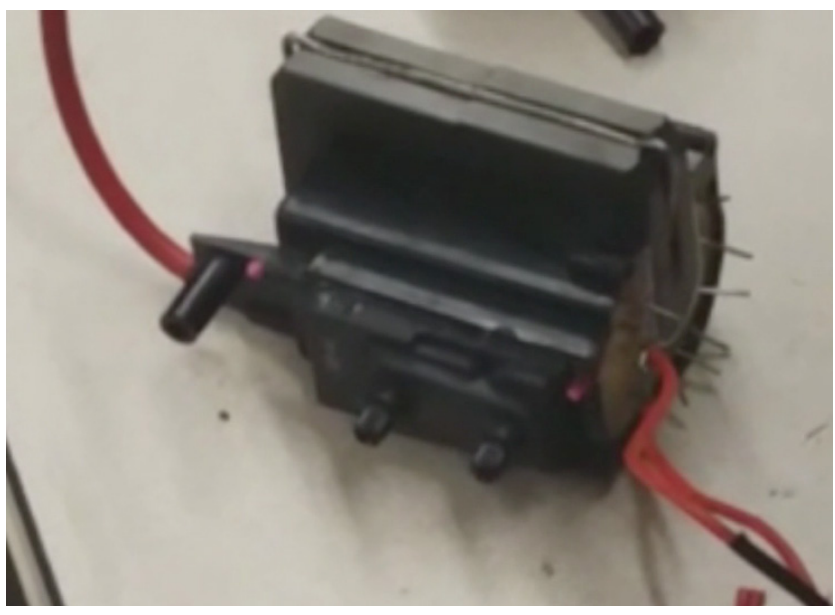

La figura 3 muestra el circuito que se utiliza para alimentar el transformador, que consta de tres partes.

Duplicador de voltaje: en el semiciclo positivo de la onda de entrada se polariza en directo uno de los diodos, que carga el condensador a la tensión pico. La polaridad es positiva (+). El otro diodo está en inverso y no conduce.

En el semiciclo negativo se polariza en directo el segundo diodo, que carga el otro condensador a la tensión pico máxima de la onda de entrada. La polaridad es negativa (-).

Los dos condensadores están en serie, las tensiones almacenadas se suman, y se logra el doble de la tensión. Este doblador de tensión es la fuente del circuito oscilador con rectificador controlado de silicio (SCR).

Figura 3. Circuito doblador de voltaje

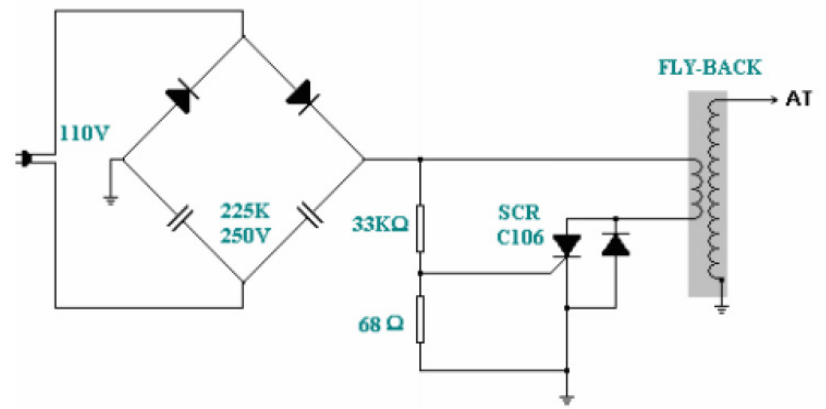

1 Para ver el flyback en funcionamiento se puede visitar esta URL https:// youtu.be/Kyq_iEATkFE (consultado el 12 de septiembre de 2017)
Oscilador de relajación: el rectificador controlado de silicio (SCR por sus siglas en inglés) descarga el condensador a través del primario del flyback cuando la tensión de la compuerta supera la tensión de disparo.

Transformador elevador de voltaje: "Los transformadores son máquinas estáticas capaces de cambiar los valores de la tensión. El transformador está formado por un núcleo de material sensible al campo magnético, con un bobinado primario conectado a un generador y un bobinado secundario conectado al circuito de utilización.

La tensión del bobinado primario V1 crea un campo magnético que recorre el circuito magnético y que induce una tensión $\mathrm{V} 2$ en el secundario. La relación entre las tensiones se llama relación de transformación y viene dada por la expresión" (Santander, s. f.):

$$
V 1 \cdot / 1=V 2 \cdot / 2
$$

La figura 4 muestra cómo el voltaje de salida de alta tensión del transformador V2 es dada por la multiplicación entre el voltaje de entrada V1 por el embobinado secundario N2 sobre el embobinado primario N1.

$$
\begin{array}{ll}
V 2 & N 2 \\
\forall 1= & N 1
\end{array}
$$

Figura 4. Diseño flyback

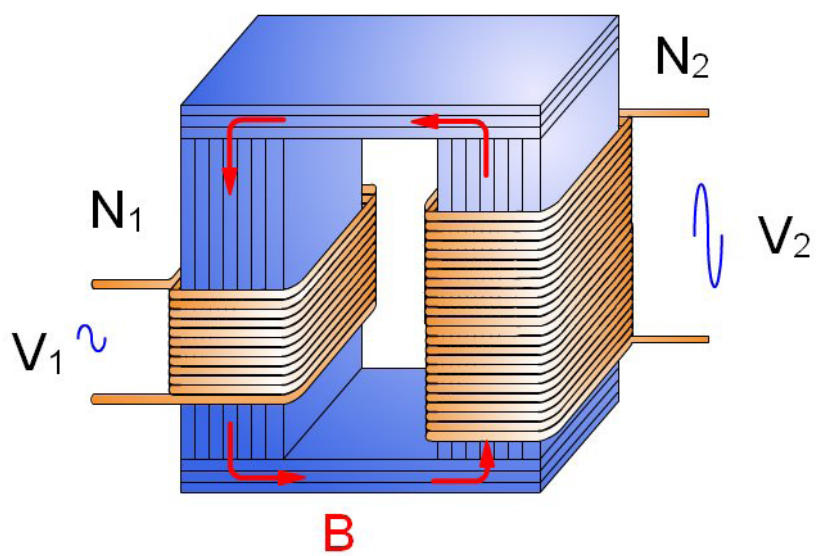

Fuente: Onuba Electrónica, s. f. 


\section{B. Fuente de contaminación}

Se escoge como fuente contaminante la quema de materiales, como la madera, carbón, papel y cigarrillos, ya que la polución de estos es la más común en cualquier lugar del mundo, incluyendo hogares e industrias, son financieramente asequibles y fáciles de manipular, y simulan situaciones perfectas, como un campo industrial o un incendio forestal.

\section{Diseño del dispositivo}

El diseño del PES se basó en uno de los tipos industriales, como se muestra en la figura 5 . El tipo de PES es de placas planas. Estas placas tienen una medida de $20 \mathrm{~cm} \times 11 \mathrm{~cm} \times 0.4 \mathrm{~cm}$. Las placas, que son enteramente de cobre, están ubicadas en parejas, excepto por las placas extremas, las cargas de las placas son intercaladas de negativo a positivo, lo que hace que las placas de carga negativa sean las de los extremos.

Figura 5. Precipitador industrial

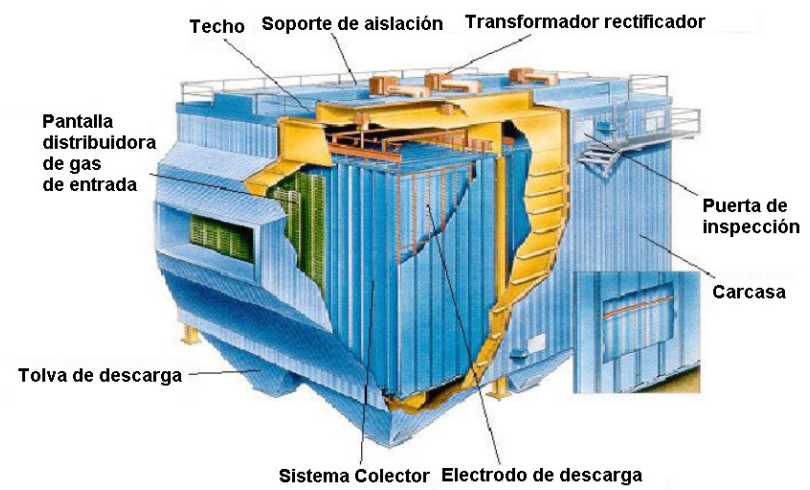

Fuente: Fernández, s. f.

Se empezó a trabajar con el circuito y el transformador para evaluar el rendimiento y la capacidad de descarga "efecto corona". Por manejar un alto voltaje, se mantuvo cierta distancia entre el cable positivo y el negativo del transformador.

Se hizo una prueba con dos placas para observar la cantidad de descargas que ocurrían en un tiempo de- terminado; a la vez, se observó cómo las placas reaccionaron con pedazos de papel quemado. ${ }^{2}$

El modelo y la distancia de las placas se basaron tanto en el campo eléctrico que crean, como en la frecuencia de las descargas entre las placas. Con base en lo anterior se empezó a trabajar en un modelo 3D, como se muestra en la figura 6 .

Figura 6. Caja de placas

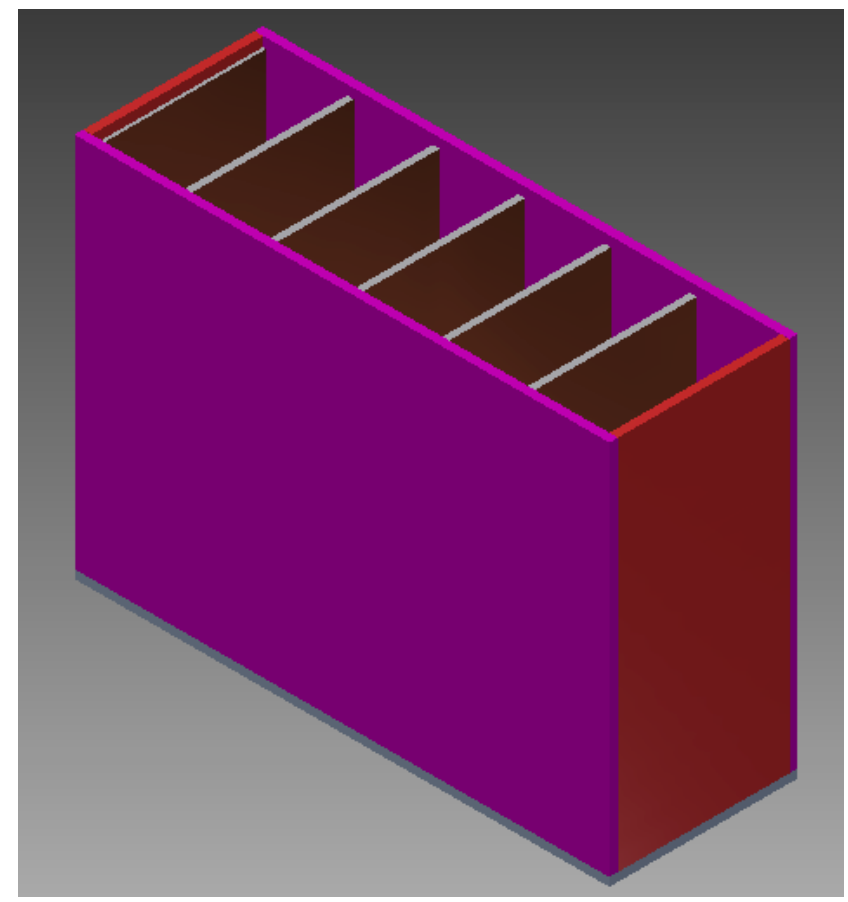

La cámara de aire (figura 7), es el lugar donde son atraídas las partículas por medio del flujo de aire que genera un ventilador. Es configurable en el interior para poder dirigir de la forma más efectiva la corriente de aire hacia las placas. En esta misma estructura se ubica uno de los sensores de gases, que mide el porcentaje del contaminante que se encuentra en este espacio antes de ser llevado hacia las placas.

2 Se pueden observar los resultados en https://youtu.be/03J8EbMQydo (consultado el 12 de septiembre de 2017). 
Figura 7. Cámara de aire

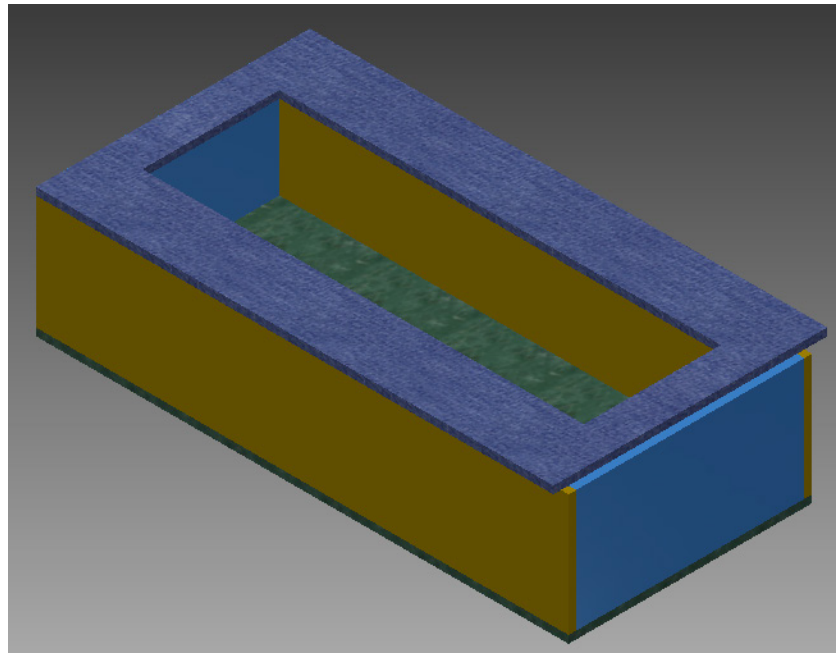

La figura y las medidas en la version de CAD se basaron en las dimensiones de las placas, debido a la necesidad de ajustarse y sostenerse de forma vertical. El material con el cual fue construido es el acrílico; de esta manera se puede observar el proceso interno de la planta. La figura 8 es el modelo del techo, en el que está ubicado uno de los sensores de gases; de esta manera se puede medir el porcentaje del contaminante que fue atrapado por el precipitador.

Figura 8. Techo del precipitador

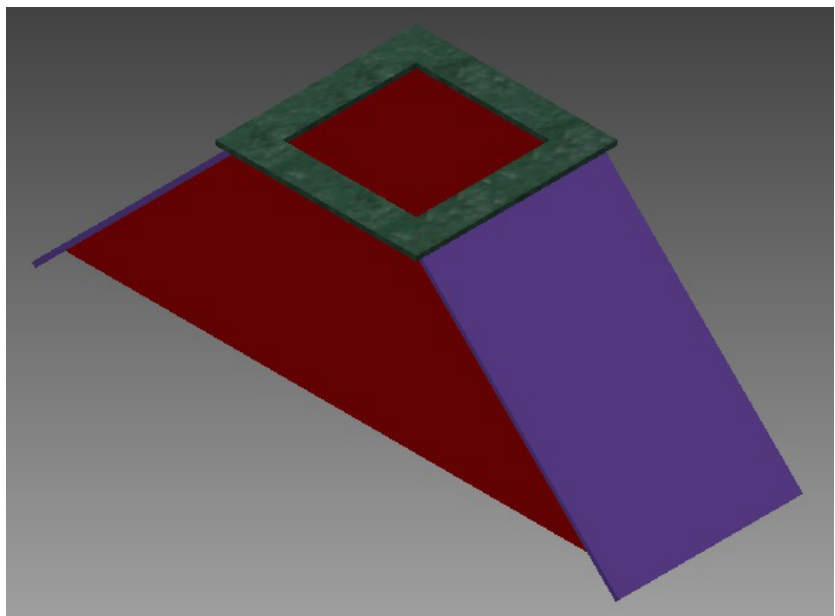

Las figuras 9, 10 y 11 muestran las partes en CAD ya cortadas y ensambladas en el material antes mencionado.
Figura 9. Precipitador ensamblado

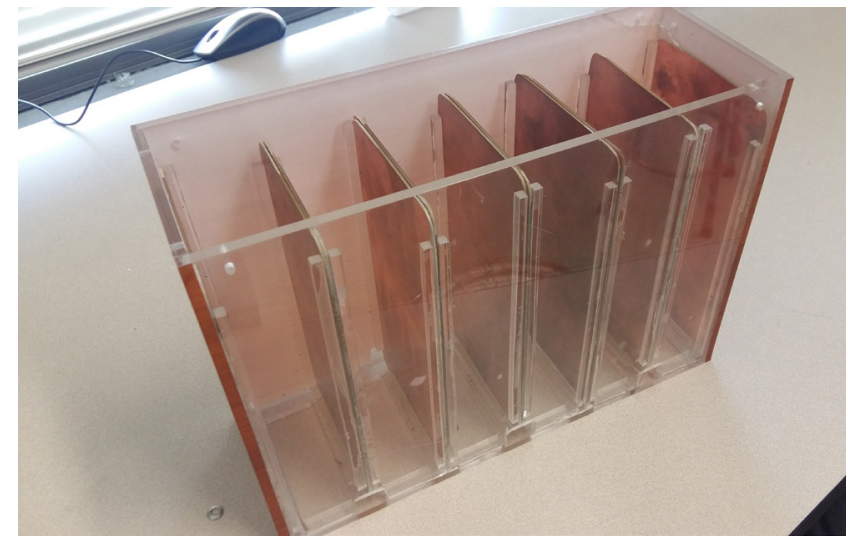

Figura 10. Cámara de aire ensamblada

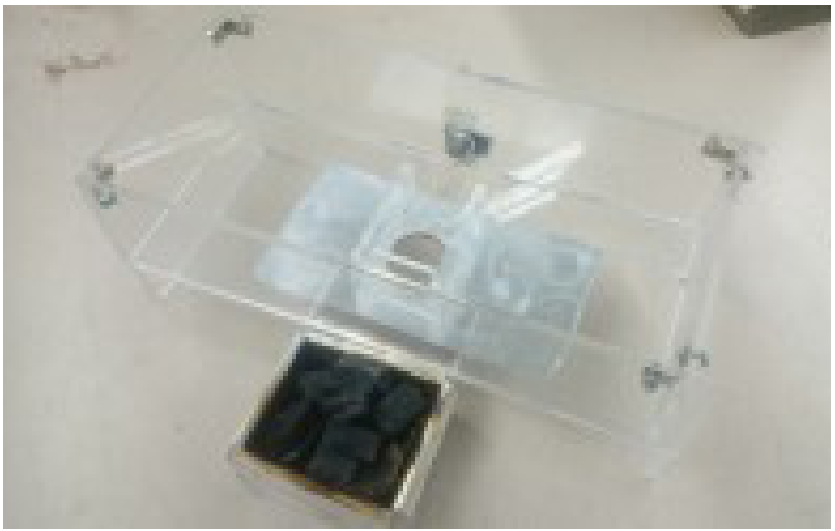

Figura 11. Techo ensamblado

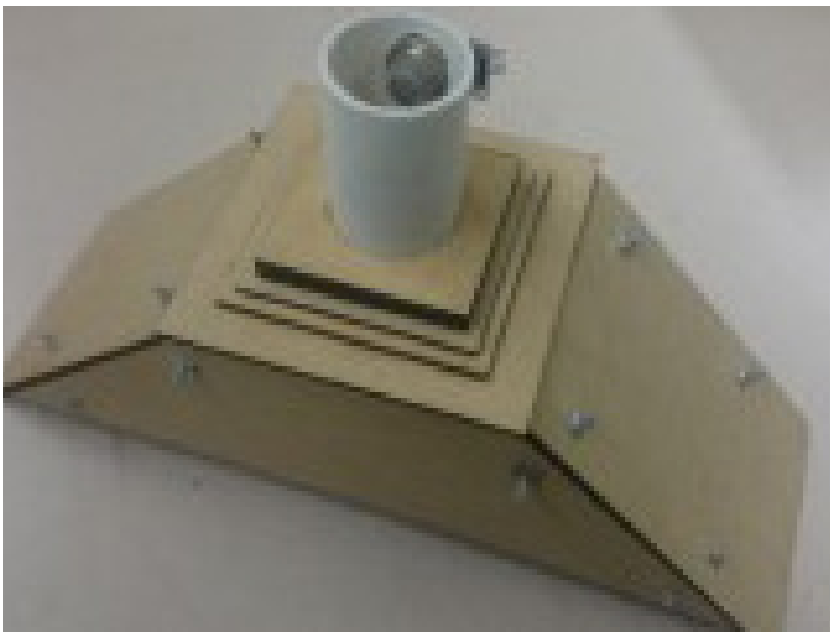




\section{RESULTADOS EXPERIMENTALES}

Para obtener resultados experimentales que permitan validar la utilidad del precipitador fue necesario diseñar un sistema de instrumentación, que fue implementado por medio de un Arduino y unos sensores que funcionan como nariz electrónica. A continuación son explicados la implementación de la instrumentación y algunos resultados experimentales. ${ }^{3}$

\section{A. Instrumentación}

Para calcular el porcentaje de partículas en la entrada y salida del sistema se implementa el uso del sensor de calidad de aire MQ-135 (figura 12). Este sensor de aire es usado para la detección de contaminación en el medio ambiente, y generalmente es implementado en circuitos de control, como alarmas en las casas, sitios donde se desea prevenir altos niveles de contaminación a nivel aeróbico, como industrias que manejan mezclas químicas que pueden ser perjudiciales para la salud, principalmente en equipos controladores de calidad de aire en edificios u oficinas. Este sensor se encarga de la detección de la densidad de gases en diversos porcentajes.

La señal de salida que proporciona este sensor es dual, de carácter analógico y digital. La señal analógica proporcionada es directamente proporcional al incremento de voltaje; en cuanto a la señal digital, presenta bajos niveles de tensión, por lo que esta puede ser procesada por un microcontrolador, como entrada o salida (esto depende de la necesidad del usuario).

Los sensores son programados mediante el uso de Arduino, que es un hardware libre que permite la programación de entradas y salidas análogas para el control de procesos o sensores, según la necesidad del usuario. El circuito consiste en conectar los pines de voltaje de los sensores a la entrada de $5 \mathrm{~V}$ del Arduino y los pines análogos van conectados a las entradas A0 y A1. Una ilustración del circuito es mostrada en la figura 13.

3 Se pueden observar los resultados en https://youtu.be/QIvPyYQM6RM (consultado el 12 de septiembre de 2017).
Figura 12. Sensor de calidad de aire (HAOYU Electronics, 2016)

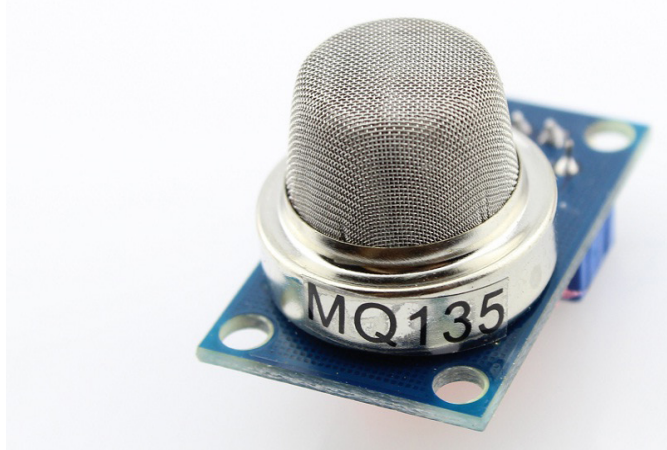

Figura 13. Circuito de sensores

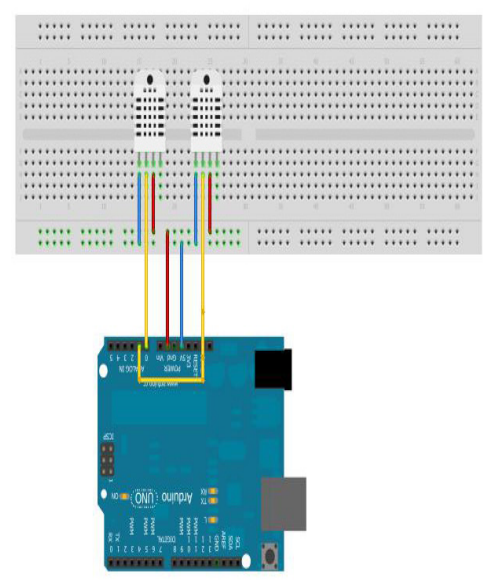

\section{B. Experimento}

Mediante el arreglo en la planta se ubican dos sensores de manera que uno esté en la cámara de aire a la entrada del contaminante "sensor 1", para así medir la concentración de este en el espacio cerrado de trabajo; el segundo sensor se ubica en la salida "sensor 2" de la planta, luego de que se realice el proceso de purificación, para de esta manera medir la concentración de partículas a la salida. Se experimentó utilizando como 
contaminante papel periódico encendido en la cámara de aire. El humo desprendido por el papel ascendía hacia las placas de cobre, que por medio del campo magnético que en ellas se generaba atrapaba gran parte de las partículas. Se observó que el humo, al salir, tenía una menor concentración que en la cámara de aire.

La figura 14 muestra el resultado del experimento ya explicado. En ella se observan dos curvas de resultados: la roja es el sensor 1, y la azul, el sensor 2. Se tomaron las muestras en un intervalo de tiempo de 0 a 13 segundos en un periodo de muestreo de $100 \mathrm{~ms}$. En el tiempo de 0 a 3,8 segundos no se aplica el contaminante, por lo cual los sensores no presentan alteraciones. En el siguiente lapso de tiempo, que fue de 3,8 a 8,9 segundos, se registró una mayor diferencia en la concentración de contaminante entre los sensores 1 y 2. En el último intervalo de tiempo, entre los segundos 8,9 y 13 , se registró una menor diferencia entre las mediciones de los sensores 1 y 2 , debido a que la fuente de contaminante ya se había consumido.

Figura 14. Datos experimentales 1

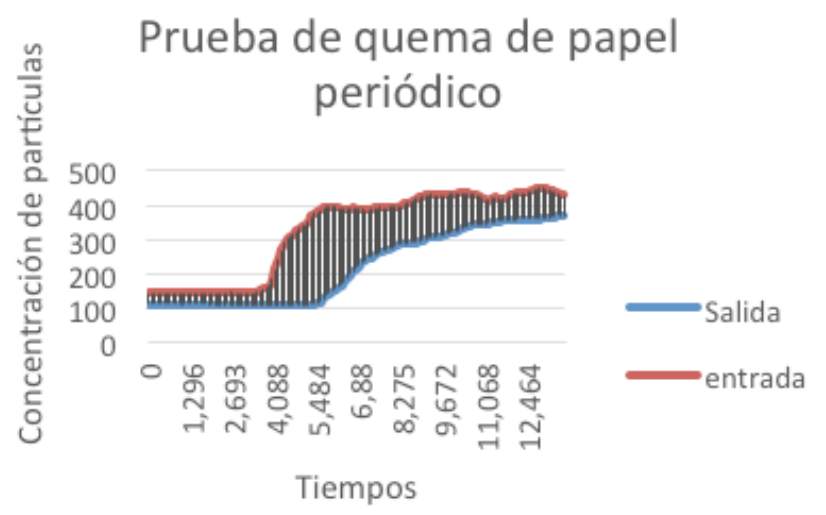

La figura 15 muestra los resultados que se tomaron quemando carbón y madera. A diferencia de los resultados de la figura 14, estos fueron realizados teniendo un ventilador en modo extractor en la salida del precipitador. Los picos observados en la fuente de alimentación ocurren principalmente por la succión del extractor, lo que demuestra que la instrumentación y el precipitador funcionan ante diferentes velocidades del flujo del aire.
Figura 15. Datos experimentales 2

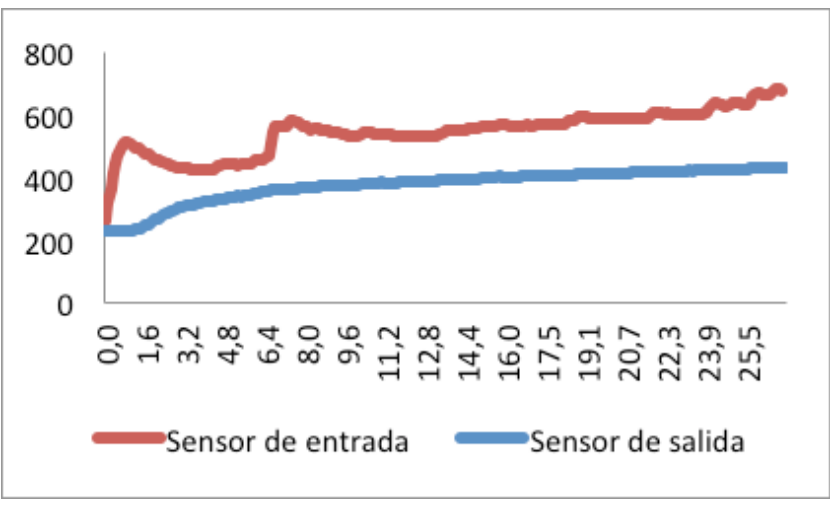

\section{CONCLUSIONES}

El prototipo de un precipitador electroestático fue implementado satisfactoriamente. Las ventajas del prototipo son los materiales de construcción, que son fáciles de conseguir y que tienen un costo de mantenimiento cercano a cero. Se aplicó el modelo de placas planas debido a que el diseño era más sencillo de construir con elementos de fácil obtención. Este prototipo se puede aplicar en contaminantes como el humo y las cenizas que produce la quema de carbón, papel, cigarrillos y madera.

Fue desarrollado un sistema de instrumentación con dispositivos de prototipado para medir los efectos del precipitador electroestático utilizando sensores de calidad del aire. Durante la experimentación pudimos resaltar que a mayor cantidad de voltaje aplicado en las placas se generaba mayor atracción de partículas, incluso partículas más pequeñas.

Trabajos futuros contemplan probar el dispositivo con una bobina de ignición y probar con otras fuentes de contaminación, como motores de vehículos.

\section{REFERENCIAS}

Cushman-Roisin, B. (2015). Notes of course introduction to environmental engineering. Disponible en http://engineering.dartmouth. edu/ d30345d/courses/engs37/esps.pdf 
Fernández, M. A. (s. f.). Seguridad y saneamento industrial. Disponible en http://managingfactory. com.ar/demo_ebooks_tecnicos/ebook_de_seg_ saneam_indi.pdf.

Fernández Sandoval, E. (2012). Mejoras en la eficiencia de los colectores de polvo tipo Jet Pulse y precipitador electrostático (tesis de grado). Piura (Perú): Universidad de Piura, Facultad de Ingeniería, p. 81.

Guerrero, D. B. (2014). Slide Share. Disponible en http://es.slideshare.net/shonEiter/produccin-decido-sulfrico.

Haoyu Electronics. (2016). Disponible en http:// www.hotmcu.com/mq135-gas-sensor-for-airqaulity-p-165.html.

Ministerio de Ambiente, Vivienda y Desarrollo Territorial (2010). Protocolo para el control y vigilancia de la contaminación atmosférica generada por fuentes fijas. Disponible en http:// www.sisaire.gov.co:8080/faces/docs/12-3-20120-25-29-818-1-1Cambios_al_Protocolo_para_el_ control_y_vigilancia_de_la_Contaminaci\%F3n_ Atmosf\%E8rica_generada_por_fuentes_fijas.pdf
Onuba Electronica (s. f.). Los transformadores. Disponible en http://www.onubaelectronica.es/ los\%20transformadores.htm.

Resolución 909 de 2008 (15 de julio). Diario Oficial, n. ${ }^{\circ} 47051$.

Santander, S. M. (s. f.). El transformador: Construcción. Disponible en https:// tecnoblogsanmartin.wordpress.com/category/ tecnologia-3\%C2\%BA-e-s-o/unidad-4energia-tecnologia-3\%C2\%BA-e-s-o/4-3-eltransformador/.

Sokhi, R. S. (2005). Air pollution control engineering. En L. Wang, N. Pereira y Y. Hung (eds.) Handbook of environmental engineering (vol. 1). New York: Humana Press.

Turner, J., Lawless, P., Yamamoto, T., Coy, D. y Research Triangle Institute (1999). Precipitadores electrostáticos. United States: Environmental Protection Agency, p. 9. 